\title{
Integration of Genomics into Rice Breeding
}

\author{
Shuichi Fukuoka • Kaworu Ebana • Toshio Yamamoto • \\ Masahiro Yano
}

Received: 8 April 2010 / Accepted: 29 June 2010/Published online: 13 July 2010

(C) Springer Science+Business Media, LLC 2010

\begin{abstract}
One of the major challenges in genetics has been to identify the nucleotide polymorphisms responsible for phenotypic variation. Through intensive analysis, several major quantitative trait loci (QTLs) for agronomic traits in rice have been identified and the underlying candidate genes have been delimited. Advanced mapping populations, including chromosome segment substitution lines, have enhanced the power of genetic analysis to detect QTL alleles, even those with minor effects. Recent examples of marker-assisted selection have proven the potential of this strategy for crop improvement. The genome-wide discovery of single nucleotide polymorphisms (SNPs), even among closely related cultivars, has enhanced the power of allele mining in a wide range of rice breeding materials. An arraybased SNP genotyping system can be used to visualize pedigree haplotypes in breeding materials, including landraces and modern cultivars. All of these technologies are accelerating the genetic dissection of complex agronomic traits and further improvement of rice.
\end{abstract}

Keywords Quantitative trait locus · Genetic mapping ·

Marker-assisted selection .

Single-nucleotide polymorphism $\cdot$ Haplotype

\section{Introduction}

Rice genomics has enhanced our understanding of the genetic control of agronomic traits and provided tools for crop

S. Fukuoka $\cdot$ K. Ebana $\cdot$ T. Yamamoto $\cdot$ M. Yano $(\square)$

QTL Genomics Research Center,

National Institute of Agrobiological Sciences,

Kannondai 2-1-2,

Tsukuba, Ibaraki 305-8602, Japan

e-mail: myano@nias.affrc.go.jp improvement. Most agronomic traits are controlled by multiple genes and show complex, quantitative inheritance. Therefore, efforts have been concentrated on identification of quantitative trait loci (QTLs) associated with beneficial traits. QTL alleles with economic value have been tagged by DNA markers and introduced into elite cultivars by marker-assisted selection (MAS). The complete sequence of the rice genome (IRGSP 2005) has supported sophisticated procedures to facilitate genetic analysis of traits of interest, and several genes for agronomic traits have been cloned (Yamamoto et al. 2009). These studies have contributed to our understanding both of the genetic control of several important traits and of the molecular mechanisms of trait expression. Additionally, recent advances in genomics technology have enabled large-scale sequencing and genotyping in rice and other organisms. These innovations have opened up possibilities for genomics-assisted breeding, a new paradigm of selection strategy in plant breeding. This paper introduces recent progress in the genetic dissection of complex traits, markerassisted introgression of agronomic traits, and whole-genome selection strategies that accelerate the genomics-assisted breeding of rice.

\section{Uncovering naturally occurring variation in complex agronomic traits}

Naturally occurring variation includes QTL alleles useful for diverse agricultural practices. In contrast to induced mutations, which often have an abnormal phenotype, QTL alleles are a source of genetic variation that is less likely to confer a penalty on agricultural productivity. In the last two decades, much attention has been paid to the genetic and molecular dissection of several important agronomic traits. Around 8,000 QTLs have been detected so far and summarized in the 
Gramene-QTL database (Jaiswal et al. 2002). Several QTLs with larger effects have been cloned by map-based strategies (Yamamoto et al. 2009); however, precise mapping of QTL alleles with small effects has been a challenge.

An excellent example of uncovering genetic control of a useful trait is the analysis of heading date, a key determinant of the adaptation of rice to different cultivation areas and cropping seasons. Several QTLs have been mapped as single Mendelian factors (Yano et al. 2001; Lin et al. 2002, 2003; Doi et al. 2004), and map-based cloning of the underlying genes has pinpointed the nucleotide polymorphisms responsible for phenotypic variation (Yano et al. 2000; Takahashi et al. 2001; Kojima et al. 2002; Doi et al. 2004; Xue et al. 2008). These studies suggest both functional similarity and divergence of genetic control between rice and Arabidopsis (Izawa 2007; Tsuji et al. 2008).

Traits associated with grain productivity are a major target for crop improvement. A QTL for semi-dwarf plant height, $s d l$, contributed to the "green revolution" in rice production and was found to contain a defective gibberellin 20-oxidase gene (Sasaki et al. 2002). Another QTL, Grain number 1a (Gnla), was identified as encoding cytokinin oxidase/dehydrogenase (Ashikari et al. 2005). These studies illustrate the important role of plant hormones in growth control and in natural trait variation. Recent studies of grain size and grain filling have identified previously uncharacterized genes for which specific alleles have been selected during crop domestication (Shomura et al. 2008; Wang et al. 2008).

Tolerance to environmental stress is an important factor for cultivated crops, enabling them to adapt to specific environments. The rice Submergence 1 (Sub1) gene encodes a putative ethylene-responsive factor that allow rice to survive complete submergence for more than 2 weeks (Xu et al. 2006). SNORKEL1 and SNORKEL2 encode transcription factors for ethylene signaling. These factors allow deep-water cultivars to elongate rapidly in response to submergence, helping the plant keep its leaves above water (Hattori et al. 2009). These finding suggest that rice has two different strategies to adapt to submergence due to flooding, both of which are regulated by accumulated ethylene inside the submerged plant. These floodingtolerant alleles have been found in limited groups of rice, supporting the idea that they may have been lost during breeding selection. This analysis offers the possibility of reintroducing such beneficial QTL alleles into rice.

Durable disease resistance has been a breeding target for many years, because most resistance genes identified so far show complete but race-specific resistance. Blast-resistance QTL allele pi21, identified from a durably resistant cultivar, encodes a proline-rich protein structurally different from previously identified resistance proteins (Fukuoka and Okuno 2001; Fukuoka et al. 2009). These studies suggest that a proline-rich motif that may have a role in proteinprotein interaction has a crucial role in the protein's function. The allele enhances the defense response and confers non-race-specific resistance, although the response against pathogen attack is not as strong as the typical resistance ( $R$-gene) response. This type of nonspecific, weaker resistance might contribute to decreased selective pressure for pathogens to overcome host resistance (Fukuoka et al. 2009).

\section{Marker-assisted enhancement of complex agronomic traits}

MAS is a promising strategy to enhance traits with economic and agricultural value in rice (Yamamoto et al. 2009). DNA markers for genes and QTLs for agronomic traits allow breeders to precisely select plants with beneficial traits in breeding programs. Recent examples of MAS address important issues in the enhancement of agronomic traits under complicated genetic control.

In one example, MAS enhanced the cropping potential of an elite cultivar by enabling the development of versions of the cultivar with diverse heading dates. Four QTLs for heading date- $H d 6, H d l, H d 4$, and $H d 5$-were introgressed from 'Kasalath' into 'Koshihikari', a leading cultivar in Japan (Takeuchi et al. 2006). The size of the introgressed chromosomal segments in those lines was very small: $300-600 \mathrm{~kb}$ in nearly isogenic lines (NILs) for $H d 1$, Hd6, and Hd5. The resulting Koshihikari NILs provide versions of the cultivar with early and late heading dates but without significant differences in agronomic performance from the original cultivar. In another example, a major QTL allele of Sub1 that confers improved submergence tolerance was backcrossed into several cultivars, providing good performance in yield and other agronomic traits as well as tolerance to submergence (Neeraja et al. 2007; Septiningsih et al. 2009). The results of these backcrosses confirmed the usefulness of this allele in submergence-prone areas in Southeast Asia.

An effective application of MAS in breeding is in the elimination of linkage drag, the co-introduction of beneficial and undesirable agricultural traits from exotic genetic resources. The durable blast resistance gene pi21 was found to be linked with gene(s) associated with inferior eating quality within a 40-kb distance (Fukuoka et al. 2009). In that study, desirable recombinants between pi21 and the genes conferring inferior eating quality were successfully selected from a large breeding population by using DNA markers for the region around pi21. The availability of DNA markers closely linked to the genes of interest enabled a breakthrough in the development of durably resistant cultivars that had not been achieved during 80 years of conventional 
breeding in Japan. This is a clear example of selection in breeding that could not have been achieved without the use of DNA markers tightly linked to the genes and DNA regions of interest.

MAS also allow us to combine beneficial QTL alleles in one plant in a relatively short time (Ashikari and Matsuoka 2006). This concept, gene pyramiding, was successfully used to develop a new high-yield line with lodging resistance in an elite genetic background. This was done by crossing an NIL containing a gene for semi-dwarfism with one containing a gene/QTL encoding increased grain number (Ashikari et al. 2005). Gene pyramiding is also useful to improve traits controlled by multiple QTLs.

\section{Allele mining for complex agronomic traits with chromosome segment substitution lines}

A key factor for success in the identification and use of QTLs for agronomic traits is the magnitude of the genetic effect of an allele relative to the effect of the environment. In some cases, a QTL has an effect large enough to be useful as a single gene. For example, the major QTL Grain number $1 a$ was cloned by using a map-based strategy; its identification contributed both to our understanding of the genetic control of spikelet development in rice and to the improvement of grain number per panicle (Ashikari et al. 2005). However, in general, a single major QTL allele is not enough to improve a trait, and additional alleles must be identified to combine with it. Information on "validated" minor QTL alleles is limited even for traits that have been characterized by many researchers (Yamamoto et al. 2009). A major reason for the difficulty in dealing with minor QTLs is the low detection power of QTL analysis using $F_{2}$ and recombinant inbred lines (RILs), owing to their highly heterogeneous genetic backgrounds.

To overcome problems associated with the limited detection power of conventional genetic mapping populations, researchers have developed chromosome segment substitution lines (CSSLs; Ebitani et al. 2005; Takai et al. 2007; Ando et al. 2008; Hori et al. 2010). In these novel plant materials, a particular chromosomal region in the recurrent parent is replaced by the corresponding segment from a donor genotype. CSSLs allow one to detect QTL alleles in the substituted region with high detection power and to efficiently survey QTLs within that part of the donor chromosome.

Genetic analysis using CSSLs provides a systematic research flow from the detection of QTLs for useful traits to their cloning and molecular breeding (Fig. 1). Once a phenotypic difference between a CSSL and the recurrent parent is identified, researchers can immediately identify the chromosomal region of that QTL, because each CSSL normally contains a single chromosomal segment from the donor. Comparison of the position of donor chromosome regions among a set of CSSLs for that chromosome enables us to further delineate the candidate chromosomal region (substitution mapping). Mapping populations and NILs for further genetic analysis of the QTL without background noise (i.e., the effect of other segregating QTLs) can be obtained readily from the progeny of a single backcross with that CSSL. Such genetic materials allow us to determine the genetic effect of a given QTL allele, even one with a small effect (Miura et al. 2001; Sato et al. 2003; Ueda et al. 2004; Ebitani et al. 2005; Ando et al. 2008; Hori et al. 2010). After confirmation of genetic effect of a QTL, map-based cloning can be started quickly from these materials, by developing populations for high-resolution mapping.

CSSLs are a powerful tool to determine phenotypic differences conferred by various QTL alleles. Sequence analysis of the pi21 blast resistance gene from a number of cultivars identified 12 alleles (based on insertion-deletion polymorphisms in the region) that might be involved with the protein's function (Fukuoka et al. 2009). However, it was sometimes difficult to infer the effect of each mutation from the sequence only. Developing CSSLs for the chromosome region of interest by using diverse germplasm as the donor material provided a solution to this problem. Evaluation of this series of CSSLs, each possessing one of the pi21 alleles in the genetic background of a susceptible cultivar, clearly demonstrated that all except one were susceptible, and suggested that two deletions in the allele are required to confer resistance (Fukuoka et al. 2009). The analysis also revealed that the resistance allele was present in some strains of japonica rice, suggesting that it could improve blast resistance of rice worldwide. This example illustrates the usefulness of information on the distribution of QTL alleles when a breeding strategy is being planned.

Another application of CSSLs is the analysis of genetic interaction. Although the power of detection of epistatic interactions in CSSLs is not as high as that in primary mapping populations such as $F_{2} \mathrm{~S}$ and RILs, CSSLs provide unique opportunities to detect interactions among chromosomal regions involved in target traits within a highly homogeneous genetic background. In previous studies, NILs containing single QTLs have been developed and then crossed to combine two or three QTLs into a single genetic background so as to clarify the epistatic interactions among the QTLs (Lin et al. 2000; Yamamoto et al. 2000; Lin et al. 2003). However, it takes much time and effort to develop NILs for all pairs of putatively interacting QTLs. In contrast, CSSLs already developed for allele mining or other purposes can be used to validate a putative epistatic interaction relatively quickly by using progeny from a cross between CSSLs containing the QTLs of interest. 


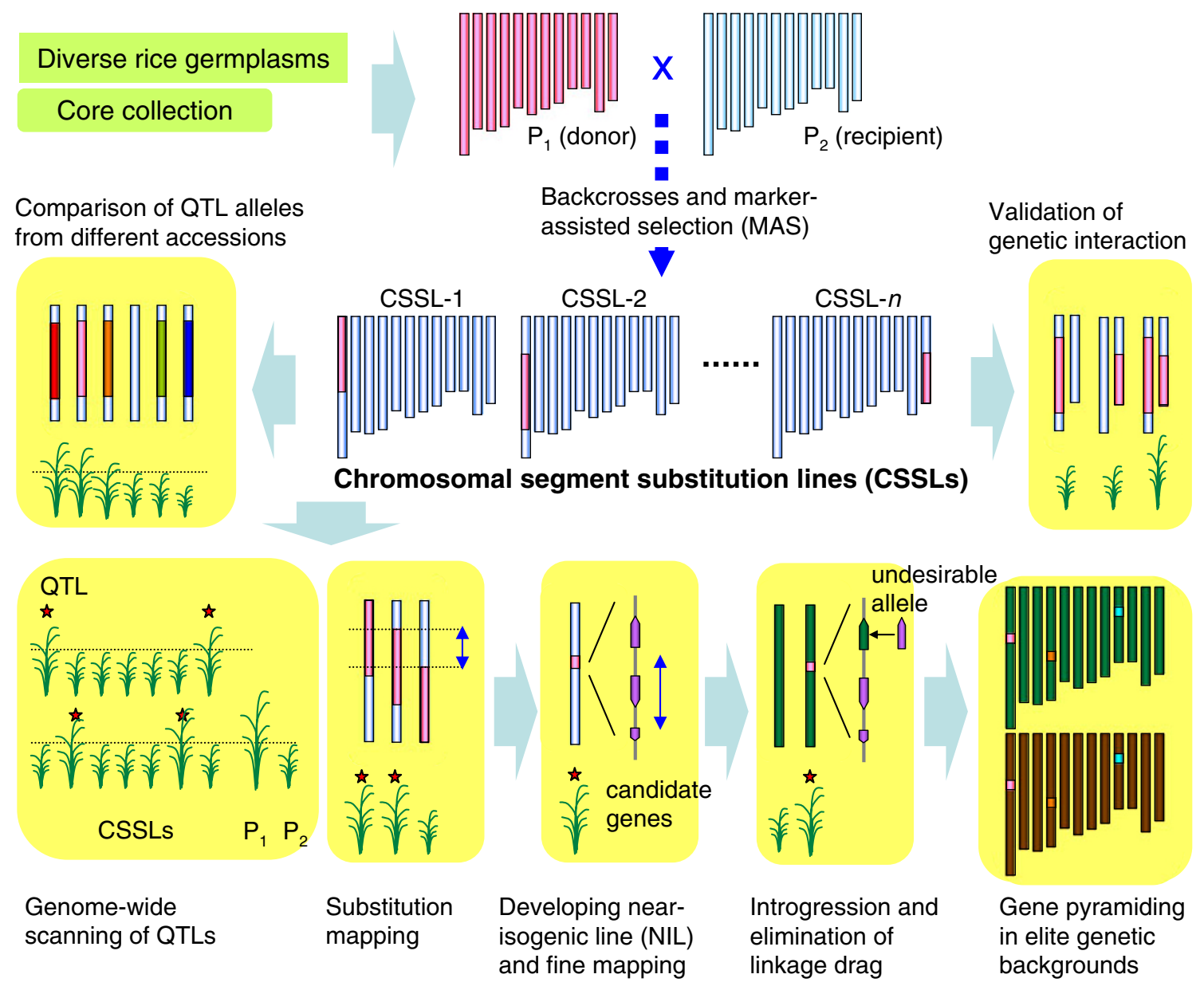

Fig. 1 Research flow from QTL mining to molecular breeding. See text for details.

The power of research using CSSLs depends on the identification and availability of donor cultivars. We analyzed the genetic structure of cultivated rice by genome-wide RFLP analysis and selected core collections that represent the genetic diversity (Kojima et al. 2005). We are now developing CSSLs from a wide range of accessions selected from our core collection as donors and Koshihikari as the recurrent parent. These materials will enhance the potential of systematic research for allele mining and explaining complex agronomic traits in rice.

\section{Genome-wide discovery of single nucleotide polymorphisms and development of genotyping arrays}

Until now, simple sequence repeats have been the major source of DNA markers used for genetic analysis and rice breeding. We believe that single nucleotide polymorphisms (SNPs) will become the main source of genetic markers, because the SNP is the most frequent type of DNA polymorphism. After release of the detailed rice genome sequence by the International Rice Genome Sequencing
Project (IRGSP 2005), SNPs between japonica 'Nipponbare' and each of two indica lines (93-11 and GLA3) were found throughout the genome (Han and Xue 2003; Feltus et al. 2004; Shen et al. 2005; Wang et al. 2009). With the technological innovations of high-throughput sequencing (Schuster 2008), SNP information from various rice cultivars will be discovered rapidly and cost-effectively. Recently, a genome-wide re-sequencing analysis of 20 diverse indica and japonica cultivars produced about 160,000 SNPs (McNally et al. 2009), which enabled us to introgress a large number of QTLs at high resolution in our rice breeding program. However, it is still unrealistic to determine SNP genotypes by wholegenome sequencing of diverse accessions, improved cultivars, and segregating progeny of the crosses used for rice breeding. Genome-wide SNP typing arrays can be used to solve this problem (Shen et al. 2005; Gupta et al. 2008), simultaneously genotyping thousands of SNPs. The use of SNPs will help to validate the effectiveness of genome-wide association mapping (GWA; Breseghello and Sorrells 2006) for exploiting natural genetic resources with economic value. 
In one application of SNP typing arrays to crop breeding, genotypes of improved barley germplasms were surveyed at 1,524 SNP loci (Rostoks et al. 2006). This study examined the extent of linkage disequilibrium (LD) and the potential of GWA among European elite barley cultivars, illustrating the challenge of associating haplotypes with phenotypes resulting from artificial selection at a genome-wide level. Although the application of GWA may be relied on the use of appropriate population and reliable trait evaluation, this study demonstrated a potential power of the connection between haplotype definition and selection in breeding program. In rice, whole-genome genotyping of rice based on SNPs between closely related elite japonica cultivars enabled the definition of 18 consensus haplotype blocks that have been inherited from ancestral landraces, and indicated that haplotype diversity has decreased during the history of rice breeding (Yamamoto et al. 2010). The accumulated haplotype information will help us to plan breeding programs and to monitor recombination events between haplotype blocks. Eventually, whole-genome SNP typing assays will facilitate efficient rice improvement as they become integrated into current MAS systems.

To obtain sufficient numbers of SNPs in the populations that breeders actually deal with, it will be necessary to evaluate the frequency of the SNPs in these populations. Our recommended strategy is (1) to conduct whole-genome sequencing of several representative cultivars in a set of accessions (selected from ecotypes identified by population structure analysis or from genetically similar breeding lines) and (2) to detect the genotypes of all the lines in the population by using SNP typing arrays. However, we do not yet know the appropriate density of SNPs to discriminate all the recombination events in breeding populations at minimum expense. A comprehensive haplotype database based on genome-wide SNPs will be needed before the typing arrays can be designed.

\section{From gene selection to genome selection}

A strategy for the integration of genomics-oriented research activities into rice breeding is summarized in Fig. 2. Currently, marker-assisted breeding is conducted by direct or indirect selection of a target allele by using DNA markers associated with the locus (upper part of Fig. 2), but a trait of interest is not always determined by a single locus. Rather, several loci with minor phenotypic effects may epistatically or cumulatively determine phenotypes. It has been difficult to deal with such genes with minor effects in MAS, but genome-wide SNP analysis will provide new solutions and tools for this problem. By using SNP analysis, we can define haplotype blocks (lower part of Fig. 2). When a haplotype includes more than one valuable allele, it is important to define a set of DNA markers corresponding to chromosomal blocks large enough to confer the desired phenotypic effect. When a haplotype causes both preferable and inferior phenotypes because of linkage drag, it needs to be split by recombination to create a new desirable haplotype. A large number of available SNPs will help to illustrate the genetic structure of haplotypes in detail. However, our ability to define such haplotypes needs to be improved. The decay length of LD

Fig. 2 Genome-assisted breeding: integration of gene selection and haplotype selection. See text for details.
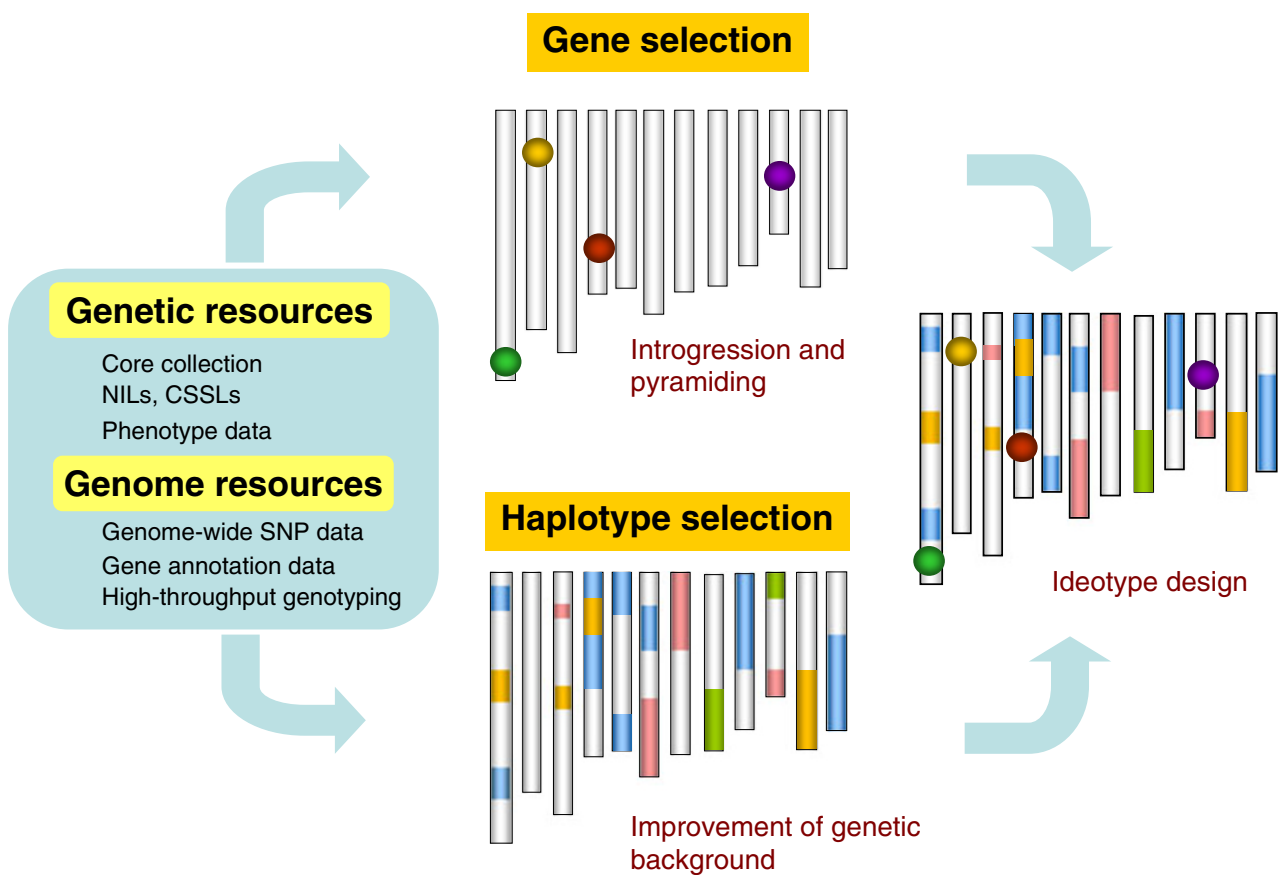
calculated among cultivars used in the analysis can offer a clue to the size of the haplotype.

To fully utilize haplotypes in breeding, additional progress will be needed in methods to associate a given haplotype with phenotypic performance. Currently, there is no systematic method to link a certain haplotype with a specific phenotype. Although GWA and genomic selection (Zhong et al. 2009) have been proposed as tools for plant genetics and breeding, the power for detecting useful alleles in such approaches and their effectiveness in breeding remain to be clarified. From the view of experimental materials, the validation of nested association mapping populations (McMullen et al. 2009) and multi-parent advanced generation inter-cross populations (Cavanagh et al. 2008) has been performed in maize and Arabidopsis, respectively. These approaches need to be practiced in rice. Those efforts will provide new criteria for selection. Finally, the major QTL alleles identified by any map-based strategy must be introgressed into preferable genetic backgrounds selected by haplotype analysis (Fig. 2). Future progress in genotype-driven rice breeding could be realized not only by gene (allele) replacement, but also by background (haplotype) improvement.

\section{Future perspectives}

MAS, developed as an armchair theory about 20 years ago, have been successful at targeting several QTLs with major effects. Technological improvements in genomics and the establishment of materials such as CSSLs for allele mining will accelerate the accumulation of information on beneficial QTL alleles and linked DNA markers. However, rice breeders need additional QTL alleles, including those with minor effects, for further improvement of this crop. With this goal in mind, the workability of a given strategy largely depends on how we deal with diverse germplasm and how we reliably phenotype materials and identify useful genes with minor effects, to integrate such information into our research flow. In the near future, genotyping will be facilitated by automated and low-cost procedures. Researchers will spend more effort on developing plant materials suitable for phenotyping and on the improvement of phenotyping skills and technologies. The combination of all these resources and information will facilitate the dissection of complex traits with agricultural importance, such as yield performance, drought tolerance, and grain quality. The output from such efforts will further enhance genomics-assisted breeding to more effectively meet future human needs.

Acknowledgements We thank members of the QTL Genomics Research Center (QTL-GRC) at NIAS for helpful discussions and suggestions in the preparation of this manuscript. Work in QTL-GRC is supported by funds (Integrated Research Project for Plant, Insect and Animals Using Genome Technology and Genomics for Agricultural Innovation, QTL-2001, NVR-0001, NVR-0002) from the Ministry of Agriculture, Forestry and Fisheries, Japan.

\section{References}

Ando T, Yamamoto T, Shimizu T, Ma XF, Shomura A, Takeuchi Y, et al. Genetic dissection and pyramiding of quantitative traits for panicle architecture by using chromosomal segment substitution lines in rice. Theor Appl Genet. 2008;116:881-90.

Ashikari M, Matsuoka M. Identification, isolation and pyramiding of quantitative trait loci for rice breeding. Trends Plant Sci. 2006;11:344-50.

Ashikari M, Sakakibara H, Lin S, Yamamoto T, Takashi T, Nishimura $\mathrm{A}$, et al. Cytokinin oxidase regulates rice grain production. Science. 2005;309:741-5.

Breseghello F, Sorrells ME. Association mapping of kernel size and milling quality in wheat (Triticum aestivum L.) cultivars. Genetics. 2006;172:1165-77.

Cavanagh C, Morell M, Mackay I, Powell W. From mutations to MAGIC: resources for gene discovery, validation and delivery in crop plants. Curr Opin Plant Biol. 2008;11:215-21.

Doi K, Izawa T, Fuse T, Yamanouchi U, Kubo T, Shimatani Z, et al. Ehdl, a B-type response regulator in rice, confers short-day promotion of flowering and controls FT-like gene expression independently of Hd1. Genes Dev. 2004;18:926-36.

Ebitani T, Takeuchi Y, Nonoue Y, Yamamoto T, Takeuchi K, Yano M. Construction and evaluation of chromosome segment substitution lines carrying overlapping chromosome segments of indica rice cultivar 'Kasalath' in a genetic background of japonica elite cultivar 'Koshihikari'. Breed Sci. 2005;55:65-73.

Feltus FA, Wan J, Schulze SR, Estill JC, Jiang N, Paterson AH. An SNP resource for rice genetics and breeding based on subspecies indica and japonica genome alignments. Genome Res. 2004;14:1812-9.

Fukuoka S, Okuno K. QTL analysis and mapping of pi21, a recessive gene for field resistance to rice blast in Japanese upland rice. Theor Appl Genet. 2001;103:185-90.

Fukuoka S, Saka N, Koga H, Ono K, Shimizu T, Ebana K, et al. Loss of function of a proline-containing protein confers durable disease resistance in rice. Science. 2009;325:998-1001.

Gupta PK, Rustgi S, Mir RR. Array-based high-throughput DNA markers for crop improvement. Heredity. 2008;101:5-18.

Han B, Xue Y. Genome-wide intraspecific DNA-sequence variations in rice. Curr Opin Plant Biol. 2003;6:134-8.

Hattori Y, Nagai K, Furukawa S, Song XJ, Kawano R, Sakakibara H, et al. The ethylene response factors SNORKEL1 and SNORKEL2 allow rice to adapt to deep water. Nature. 2009;460:1026-30.

Hori K, Sugimoto K, Nonoue Y, Ono N, Matsubara K, Yamanouchi $\mathrm{U}$, et al. Detection of quantitative trait loci controlling pre-harvest sprouting resistance by using backcrossed populations of japonica rice cultivars. Theor Appl Genet. 2010;120:1547-57.

IRGSP. The map-based sequence of the rice genome. Nature. 2005;436:793-800.

Izawa T. Day length measurements by rice plants in photoperiodic short-day flowering. Int Rev Cytol. 2007;256:191-222.

Jaiswal P, Ware D, Ni J, Chang K, Zhao W, Schmidt S, et al. Gramene: development and integration of trait and gene ontologies for rice. Comp Funct Gen. 2002;3:132-6.

Kojima S, Takahashi Y, Kobayashi Y, Monna L, Sasaki T, Araki T, et al. $H d 3 a$, a rice ortholog of the Arabidopsis FT gene, promotes transition to flowering downstream of $H d l$ under short-day conditions. Plant Cell Physiol. 2002;43:1096-105. 
Kojima Y, Ebana K, Fukuoka S, Nagamine T, Kawase M. Development of an RFLP-based rice diversity research set of germplasm. Breed Sci. 2005;55:431-40.

Lin HX, Yamamoto T, Sasaki T, Yano M. Characterization and detection of epistatic interactions of 3 QTLs, $H d 1$, $H d 2$, and $H d 3$, controlling heading date in rice using nearly isogenic lines. Theor Appl Genet. 2000;101:1021-8.

Lin HX, Ashikari M, Yamanouchi U, Sasaki T, Yano M. Identification and characterization of a quantitative trait locus, $H d 9$, controlling heading date in rice. Breed Sci. 2002;52:35-41.

Lin HX, Liang ZW, Sasaki T, Yano M. Identification and characterization of a quantitative trait locus, $H d 4$ and $H d 5$, controlling heading date in rice. Breed Sci. 2003;53:51-9.

McMullen MD, Kresovich S, Villeda HS, Bradbury P, Li H, Sun Q, et al. Genetic properties of the maize nested association mapping population. Science. 2009;325:737-40.

McNally KL, Childs KL, Bohnert R, Davidson RM, Zhao K, Ulat VJ, et al. Genomewide SNP variation reveals relationships among landraces and modern varieties of rice. Proc Natl Acad Sci USA. 2009; 106:12273-8.

Miura K, Lin SY, Yano M, Nagamine T. Mapping quantitative trait loci controlling low temperature germinability in rice (Oryza sativa L.). Breed Sci. 2001;51:293-9.

Neeraja CN, Maghirang-Rodriguez R, Pamplona A, Heuer S, Collard $\mathrm{BC}$, Septiningsih EM, et al. A marker-assisted backcross approach for developing submergence-tolerant rice cultivars. Theor Appl Genet. 2007;115:767-76.

Rostoks N, Ramsay L, MacKenzie K, Cardle L, Bhat PR, Roose ML, et al. Recent history of artificial outcrossing facilitates wholegenome association mapping in elite inbred crop varieties. Proc Natl Acad Sci USA. 2006;103:18656-61.

Sasaki A, Ashikari M, Ueguchi-Tanaka M, Itoh H, Nishimura A, Swapan D, et al. Green revolution: a mutant gibberellin-synthesis gene in rice. Nature. 2002;416:701-2.

Sato T, Ueda T, Fukuta Y, Kumagai T, Yano M. Mapping of quantitative trait loci associated with ultraviolet-B resistance in rice (Oryza sativa L.). Theor Appl Genet. 2003;107:1003-8.

Schuster SC. Next-generation sequencing transforms today's biology. Nat Methods. 2008;5:16-8.

Septiningsih EM, Pamplona AM, Sanchez DL, Neeraja CN, Vergara GV, Heuer S, et al. Development of submergence-tolerant rice cultivars: the Sub1 locus and beyond. Ann Bot. 2009;103:15160.

Shen R, Fan JB, Campbell D, Chang W, Chen J, Doucet D, et al. High-throughput SNP genotyping on universal bead arrays. Mutat Res. 2005;573:70-82.

Shomura A, Izawa T, Ebana K, Ebitani T, Kanegae H, Konishi S, et al. Deletion in a gene associated with grain size increased yields during rice domestication. Nat Genet. 2008;40:1023-8.

Takahashi Y, Shomura A, Sasaki T, Yano M. Hd6, a rice quantitative trait locus involved in photoperiod sensitivity, encodes the alpha subunit of protein kinase CK2. Proc Natl Acad Sci USA. 2001;98:7922-7.

Takai T, Nonoue Y, Yamamoto S, Yamanouchi U, Matsubara K, Liang ZW, et al. Development of chromosome segment substitution lines derived from backcross between indica donor rice cultivar 'Nona Bokra' and japonica recipient cultivar 'Koshihikari'. Breed Sci. 2007;57:257-61.

Takeuchi Y, Ebitani T, Yamamoto T, Sato H, Ohta H, Hirabayashi H, et al. Development of isogenic lines of rice cultivar Koshihikari with early and late heading by marker-assisted selection. Breed Sci. 2006;56:405-13.

Tsuji H, Tamaki S, Komiya R, Shimamoto K. Florigen and the photoperiodic control of flowering in rice. Rice. 2008;1:25-35.

Ueda T, Sato T, Numa H, Yano M. Delimitation of the chromosomal region for a quantitative trait locus, $q U V R-10$, conferring resistance to ultraviolet-B radiation in rice (Oryza sativa L.). Theor Appl Genet. 2004;108:385-91.

Wang E, Wang J, Zhu X, Hao W, Wang L, Li Q, et al. Control of rice grain-filling and yield by a gene with a potential signature of domestication. Nat Genet. 2008;40:1370-4.

Wang L, Hao L, Li X, Hu S, Ge S, Yu J. SNP deserts of Asiancultivated rice: genomic regions under domestication. J Evol Biol. 2009;22:751-61.

$\mathrm{Xu} \mathrm{K}, \mathrm{Xu} \mathrm{X}$, Fukao T, Canlas P, Maghirang-Rodriguez R, Heuer S, et al. Sub1A is an ethylene-response factor-like gene that confers submergence tolerance to rice. Nature. 2006;442:705-8.

Xue W, Xing Y, Weng X, Zhao Y, Tang W, Wang L, et al. Natural variation in Ghd7 is an important regulator of heading date and yield potential in rice. Nat Genet. 2008;40:761-7.

Yamamoto T, Lin H, Sasaki T, Yano M. Identification of heading date quantitative trait locus $H d 6$ and characterization of its epistatic interactions with $H d 2$ in rice using advanced backcross progeny. Genetics. 2000;154:885-91.

Yamamoto T, Yonemaru J, Yano M. Towards the understanding of complex traits in rice: substantially or superficially? DNA Res. 2009; 16:141-54.

Yamamoto T, Nagasaki H, Yonemaru J, Ebana K, Nakajima M, Shibaya $\mathrm{T}$, et al. Fine definition of the pedigree haplotypes of closely related rice cultivars by means of genome-wide discovery of singlenucleotide polymorphisms. BMC Genomics. 2010;11:267.

Yano M, Katayose Y, Ashikari M, Yamanouchi U, Monna L, Fuse T, et al. $H d l$, a major photoperiod sensitivity quantitative trait locus in rice, is closely related to the Arabidopsis flowering time gene CONSTANS. Plant Cell. 2000;12:2473-84.

Yano M, Kojima S, Takahashi Y, Lin H, Sasaki T. Genetic control of flowering time in rice, a short-day plant. Plant Physiol. 2001;127:1425-9.

Zhong S, Dekkers JC, Fernando RL, Jannink JL. Factors affecting accuracy from genomic selection in populations derived from multiple inbred lines: a barley case study. Genetics. 2009;182:355-64. 